\title{
Study on failure mechanical characteristics and damage constitutive model of sandstone with different numbers of holes
}

\author{
Chao Peng ${ }^{\mathrm{a}} \mathbb{D}$, Wanrong Liu ${ }^{\mathrm{b} *}$ \\ a School of Emergency Management and Safety Engineering, China University of Mining \& Technology (Beijing), Haidian, Beijing 100083, \\ China. Email: pengchaolwr@163.com \\ b Liaocheng University, Liaocheng 252059, China. Email: wanrongliu1989@163.com \\ *Corresponding author
}

http://dx.doi.org/10.1590/1679-78256052

\begin{abstract}
The existence of holes in rock is one of the key factors for the instability of underground rock mass engineering. Combined with acoustic emission equipment, uniaxial compression tests of rock specimens with different numbers of holes in the rock were carried out. The results show that with the number of holes increases, the value of peak strength and elastic modulus of the rock decrease. The acoustic emission (AE) counts gone through stable, slow increase, a sudden increase and towards zero four stages and there are corresponding to the initial compaction stage, elastic stages, strain hardening stage and post-peak strain softening stage of the stress-strain curves approximately. Based on the AE events, damage variables of rocks were obtained. The damage evolution process of rock can also be divided into three parts, including stable damage stage, slowly increasing damage stage and sharply increasing damage stage. According to Duncan model, the damage variable is introduced to obtain the rock damage constitutive model. No matter it is the pre-peak initial compaction stage, the linear elasticity stage, the strain hardening stage or the post-peak strain softening stage, the fitting curves are in good agreement with the test curves. It also illustrates the rationality of the damage constitutive model.
\end{abstract}

\section{Keywords}

Rock; holes; crack; acoustic emission; damage constitutive model

\section{Graphical Abstract}
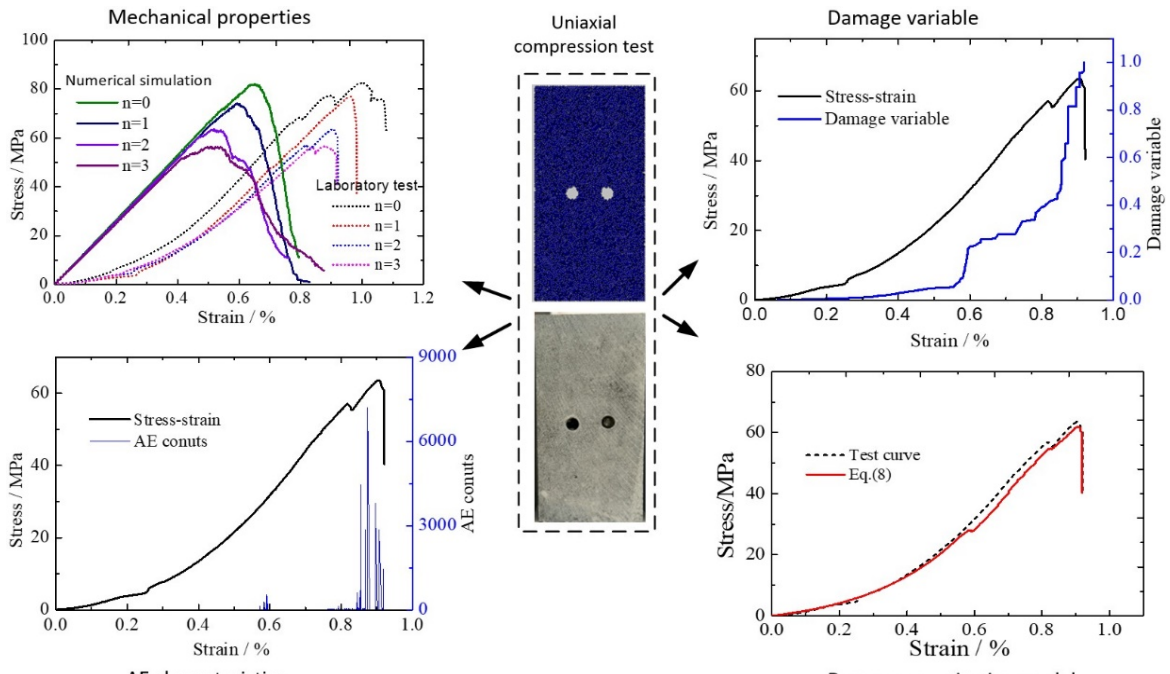

Damage constitutive model 


\section{INTRODUCTION}

The rock mass is a naturally formed heterogeneous brittle material. Due to the influence of geological structure, the rock mass contains weak structures such as rich cracks and holes. The existence of these weak structures results in obvious differences in the physical and mechanical properties of the rock mass. As a typical weak structure, holes have a significant effect on rock mass strength. A large number of studies have shown that the destruction of rock masses is also related to the holes in the rock. The holes defects in the rock mass often lead to frequent occurrence of rock mass engineering disasters. Therefore, grasping the law of the influence of holes on rock failure characteristics has important theoretical and practical significance for guiding engineering practice.

A large number of domestic and foreign scholars have done a lot of research on rock mechanics with holes. Huang et al. (2017) studied the failure characteristics of granite with pre-existing holes and crack propagation mechanism by experiments, combined with the particle flow model to further analyze the crack propagation rules around the holes of granite specimens, and analyzed the effect of holes on crack growth of granite. Lin et al. (2015) analyzed the propagation and coalescence of cracks around holes in granite with multiple holes under uniaxial compression by experimental. Research shows that granite failure is mainly affected by the number of holes coalescence. Fakhimi and Gharahbagh (2011) studied the influence of the size and distribution location of holes on the characteristics of rock failure by using discrete elements in view of the existence of holes of different sizes in actual engineering. The influence of holes on the elastic modulus of rocks and other mechanical characteristics is obtained. Wang et al. (2013) investigated the crack propagation mechanism of rock failure by water pressure in pores. It is found that the tensile fracture of rocks is affected by pore, and the existence of pore leads to a significant decrease in the tensile strength of rocks. And the pore water pressure also plays an important role in the crack propagation of rocks. Liu and Ostadhassan (2017) conducted multi-scale fractal research on shale with pores. The results show that the size and shape of the pores affect not only the strength of the rock but also the fractal law. These studies mainly analyze the propagation of cracks around the holes, there are few studies on the influence of the number of holes on the mechanical properties of rocks.

At present, AE test is one of the effective methods to monitor the progressive damage of rocks. In field engineering, Hirata et al. (2007) and Liu et al. (2015) used AE tests to evaluate the stability of the rock mass. It can be seen that acoustic emission monitoring for rock mass stability has been widely used. Moradian et al. (2010) used AE tests to study the shear damage failure process of jointed rock masses. Furthermore, the acoustic emission characteristic curve of jointed rock mass damage during the experiment can be divided into four stages. In 2015, Kim et al. (2015) conducted a compression test on brittle rocks, and evaluated the damage degree of the rocks through stress-strain curves and acoustic emission characteristics. By means of PFC software, according to the characteristics of rock scale effects, Liu et al. (2019a) conducted multi-scale experiments such as different height-diameter ratios and different widthdiameter ratios through particle discrete elements. The results show that both the mechanical characteristics and the failure morphology of the rocks are affected by the scale effect, and the scale effect of the rock must be considered when conducting rock tests. It can be seen that acoustic emission characteristics have been maturely applied in rock mechanics(Guo, Q., et al.,2020a). It is a very important monitoring method to evaluate rock damage.

Based on previous studies, this paper conducts uniaxial compression tests on rocks with different numbers of holes. Firstly, the effect of holes on the mechanical characteristics of rock failure is analyzed, then the AE events of rock with different number holes are analyzed, and finally the damage constitutive model of rock with holes is further analyzed. These studies are of great significance for understanding the damage of rock masses.

\section{UNIAXIAL COMPRESSION EXPERIMENTAL OF ROCK}

\subsection{Experimental Plan}

Holes have a great influence on the mechanical properties of rocks. In order to study the influence of the number of holes on the mechanical characteristics of rocks, uniaxial compression mechanical tests on rock specimens with different numbers of holes were carried out, and the acoustic emission events were monitored during the experimental. In order to reduce the discreteness of the test, the rock specimens were processed from sandstone with compactness and good mechanical characteristics. The color of the sandstone is gray, composed of quartz particles, high cementation degree, and good macroscopic uniformity. Sandstone specimens are cut and polished to ensure that the flatness of each section of the specimen is $0.1 \mathrm{~mm}$. And the adjacent surfaces of the specimens should be kept as vertical as possible, and finally processed into different numbers of holes rock specimens as shown in Fig.1. The size of the specimens is $50 \mathrm{~mm}$ (length) $\times 100 \mathrm{~mm}$ (height) $\times 50 \mathrm{~mm}$ (width), the hole radius is $3 \mathrm{~mm}, \mathrm{n}$ represents the number of holes. When $\mathrm{n}$ is $0,1,2,3$, 
it means intact rock, one hole, two holes and three holes, respectively. The specific hole positions and the positional relationship between the holes are shown in Figure 1. The intact rock specimens is the control group test.

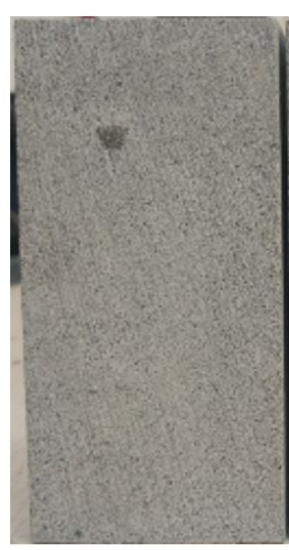

(a) $n=0$

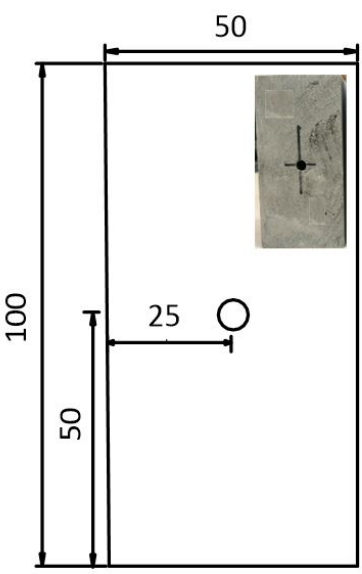

(b) $n=1$

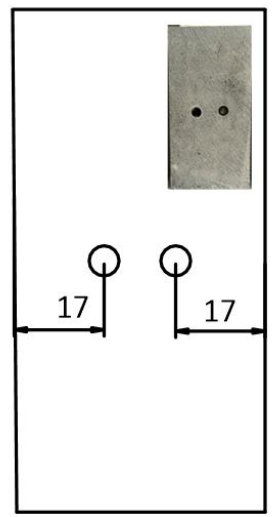

(c) $n=2$

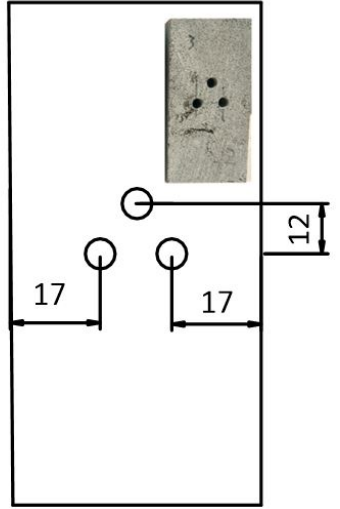

(d) $n=3$

Figure 1. Rock specimens with different number of holes (units: $\mathrm{mm}$ )

\subsection{Experimental Procedure}

The uniaxial compression loading scheme was adopted and the MTS system was used to carry out these experiments. In the experiment, the loading was controlled by displacement, and the loading rate was $0.004 \mathrm{~mm} / \mathrm{s}$, which was guaranteed to be quasi-static. In addition, the DS2 full information acoustic emission signal analyzer produced by Beijing Soft Island Technology Co., Ltd was adopted to monitor the AE characteristics of these rock specimens during the experiments. The acoustic emission probe model is RS-54A, the monitoring frequency range is $100-900 \mathrm{kHz}$, and the diameter of the probe is $8 \mathrm{~mm}$. The test equipment and process diagram is shown in Fig.2.

The specific experiment process are as follow. Firstly, the acoustic emission probes are smeared with appropriate lubricant and then placed on the rock. Secondly, the rock specimens are put on the test bench and appropriate lubricant are smeared on the surface of the models to reduce the friction effect between the loading unit and the end of the models during the test. The acoustic emission probe is arranged a little above the center of the specimen surface, as shown in Figure 2. Thirdly, the axial force is applied slowly by displacement control mode $(0.004 \mathrm{~mm} / \mathrm{s})$. The stress-strain and acoustic emission data are recorded in the test until the specimen is destroyed. The failure mode of rock specimens are photographed. Acoustic emission experiments of different rock specimens are completed by the same steps.

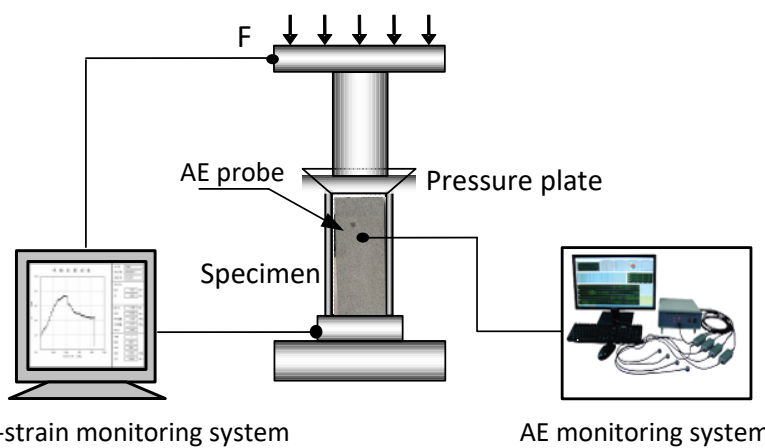

Figure 2. Test equipment and process diagram

\section{ANALYSIS OF TEST RESULTS}

\subsection{Analysis of Rock Mechanical Characteristics}

Fig. 3 and Fig. 4 are stress-strain curves and peak strength curves of rock with different number of holes. It can be seen from Fig. 3 that the stress-strain curves can be divided into 4 stages. Take the intact rock specimen as an example for analysis, and the failure process of other specimens is similar. In the initial compaction stage (OA), with the increase 
of axial stress, the micro-cracks existing in the rock are compacted under pressure, and the stress-strain curve shows a concave upward trend. At this stage, the axial strain of the rock is $0-0.4 \%$, the lateral strain is small, and the specimen volume strain decreases with increasing load. Elastic stage $(A B)$ : As the axial stress increases, the axial strain increases, and the stress-strain curve changes approximately linearly. The axial strain range is about $0.4 \%-0.8 \%$. Due to the increase of stress, cracks began to appear in the rock specimens and their growth was stable. It is also the stage of stable crack development. Strain hardening stage $(B C)$ : As the strain increases, the stress also increases but the rate of increase gradually decreases, and the stress-strain curve is convex. The maximum stress peak is $82.5 \mathrm{MPa}$, and the peak strain corresponding to the peak stress is $1.02 \%$. The yield stress is $68.1 \mathrm{MPa}$. At this stage, the cracks spread instably, a large number of cracks accumulated, and the curve fluctuated twice. Post-peak strain softening stage (CD): As the strain increases, the bearing capacity of the rock decreases rapidly, a large number of cracks penetrate through to form the failure zone, and the rock eventually failure.

It can be seen from Fig. 4 that the peak strength of rock decreases with the increase of the number of holes. When the $n$ increases from 0 to 3, the uniaxial compressive strength of rock is $82.55 \mathrm{MPa}, 76.85 \mathrm{MPa}, 63.51 \mathrm{MPa}$, and 56.61MPa respectively. The peak intensity is reduced by $31.4 \%$. The decreasing degree of uniaxial compressive strength of rock is approximately one-dimensional linear relationship with the $n$. The curve fitting accuracy value $R^{2}$ is 0.9637 . The fitting equation is as follows.

$Y=-9.11 X+83.55$

Where $\mathrm{Y}$ represents the uniaxial compressive strength of rock specimen with different $n$.

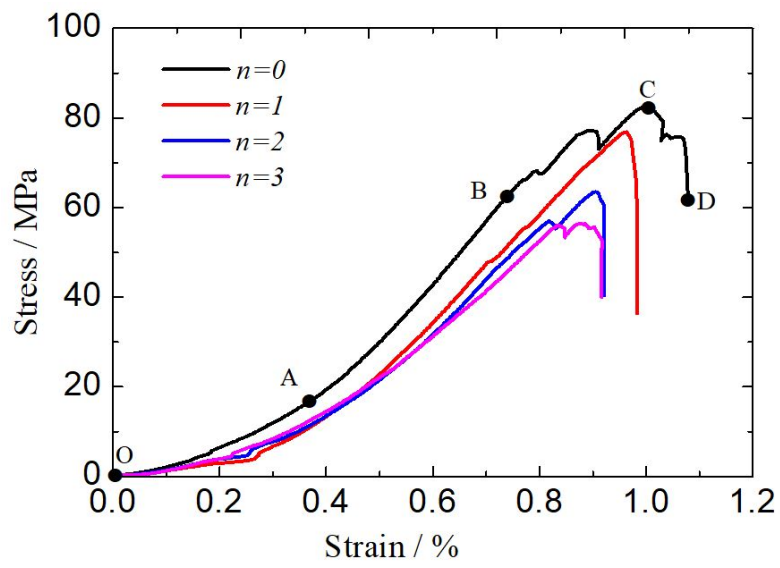

Figure 3. Stress-strain curves of rock

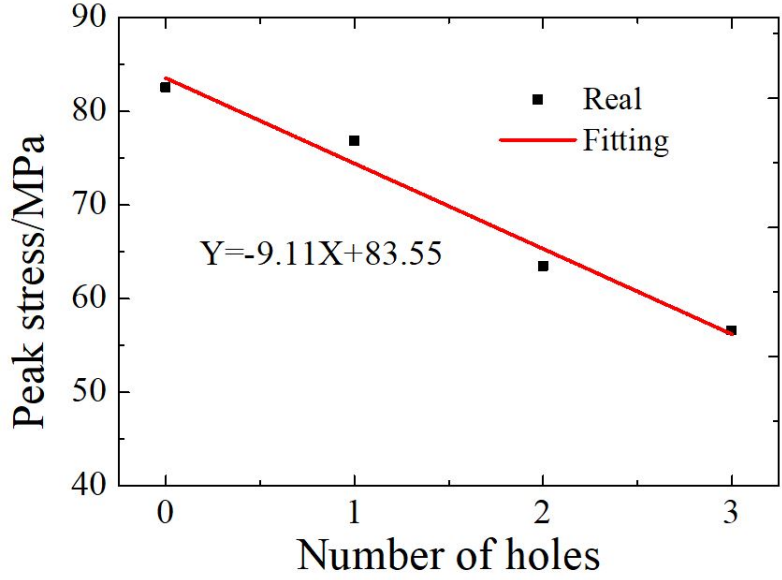

Figure 4. Peak strength curves of rock

Fig. 5 is elastic modulus curves of rock with different number of holes. It can be seen from Fig. 5 that the elastic modulus of rock decreases with the increase of the number of holes. The elastic modulus of the rock specimens are 14.17GPa, 12.72GPpa, 11.82GPa and 11.21GPa respectively as the $n$ increases from 0 to 3 . The elastic modulus is reduced 
by $20.9 \%$. It can be seen that when there are a large number of holes in the rock, the values of mechanical parameter of the rock are significantly reduced, which is also the essential reason for the easy destruction of many porous rock mass. The decreasing degree of elastic modulus of rock is approximately one-dimensional linear relationship with the $n$. The curve fitting accuracy value $\mathrm{R}^{2}$ is 0.9456 . The fitting equation is as follows.

$\mathrm{Y}=-0.978 \mathrm{X}+13.94$

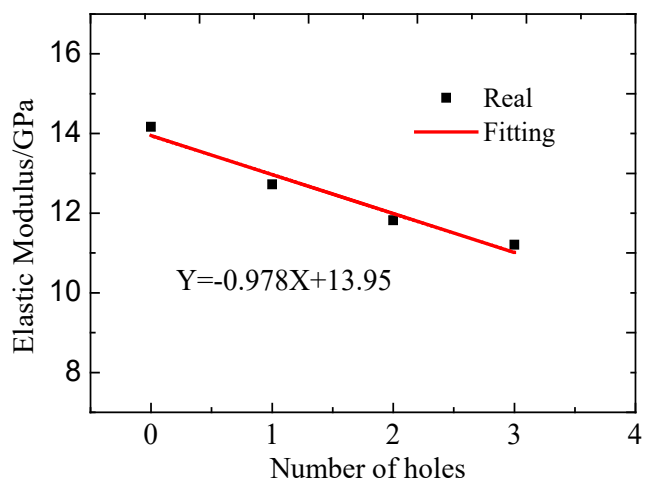

Figure 5. Elastic modulus curves of rock with different number of holes

\subsection{Analysis of Rock Failure Modes}

Fig. 6 is failure modes of rock with different number of holes. When $n$ is 0 , the failure zone formed by tensile cracks extending through the specimen at an inclination of $10^{\circ}$ results in sandstone failure. Block spalling also occurs in the lower left and upper right corners of the specimen. When $n$ is 1 , due to the existence of the hole, the upper and bottom of the rock hole are subject to tensile stress, which causes the rock to first appear tensile cracks on the upper and bottom of the hole, and then the crack extends upward and downward along the specimen, and finally causes the specimen to lose its bearing capacity. An upward tensile failure is formed above the hole, and a mixed tensile and shear failure mode is formed below the hole. When $n$ is 2 , there is a rock bridge between the two holes, and the distance between the holes is the length of the rock bridge. Due to the two holes are horizontally symmetrically arranged, the rock bridge between the two holes is not break. Under axial stress, cracks first appeared around the hole on the right side of the rock, and the cracks extended upward and downward, causing the specimen to failure. When $n$ is 3 , due to the superposition of the three hole stresses, cracks first appeared around the holes on the right side of the rock. Then the crack expand towards the upper hole, causing the rock bridge between the right and upper holes to break. Finally, the crack continued to expand in the direction of $45^{\circ}$ to the upper left and lower right corners, and eventually the crack penetrated the entire specimen and caused rock failure. Formed a mixed failure mode of tension and shear.

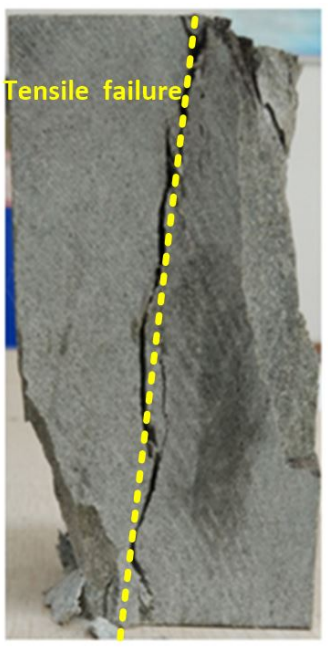

(a) $n=0$

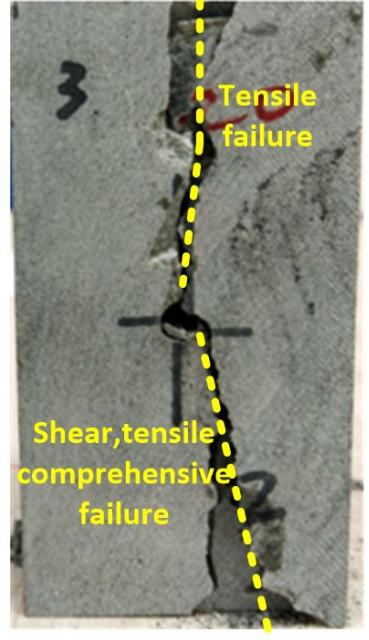

(b) $n=1$

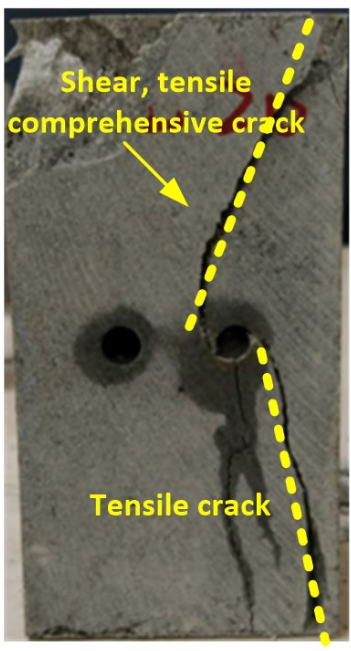

(c) $n=2$

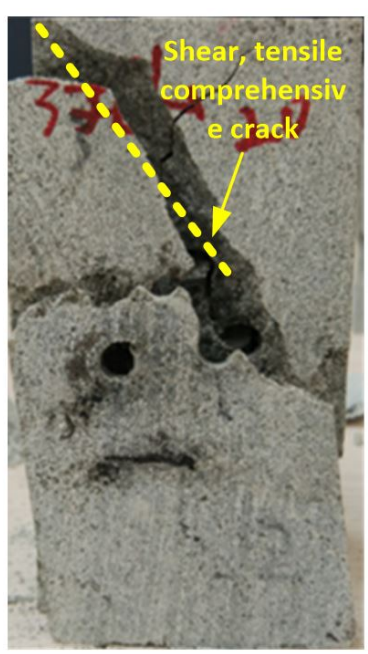

(d) $n=3$

Figure 6. Failure modes of rock with different number of holes 


\subsection{Rock AE Characteristics}

Acoustic emission tests can be characterized by many parameters, such as amplitude, energy, ringing, event count, and duration. Lockner (1993) used energy; Wasantha et al., (2014) and Wang et al., (2018) used event count. This paper use count to analyze the characteristics of $A E$ of rock.

Fig. 7 shows the stress-acoustic emission characteristic curves of the rock specimen with different number of holes. It can be seen that the change characteristics of $A E$ counts are related to the change of stress curves. The AE counts gone through stable, slow increase, a sudden increase, and towards zero four stages and these four stages are corresponding to the initial compaction stage, elastic stages, strain hardening stage, and post-peak strain softening stage of the stress curves approximately. In the initial compaction stage, there are no cracks, and there are few acoustic emission events, which are basically zero. In elastic stages, a certain number of acoustic emission events occurred, and the total number of acoustic emission events increased steadily and slowly. In strain hardening stage, acoustic emission events increased by leaps and bounds, and the total number of acoustic emission events increased sharply. In the post-peak strain softening stage, the brittle failure occurred in the rock, the stress dropped instantly, and there was almost no acoustic emission event. When the stress curve is fluctuates (e.g. Fig. 7a, b, and d), the AE count jumps suddenly at the fluctuation. This is because in the stress fluctuation place, the damage crack is produced and the release of crack energy produces acoustic emission.

The maximum value of acoustic emission counts occurs at the peak stress, and the maximum value of acoustic emission counts produced by the rock model with different number of holes is different. The maximum value of acoustic emission counts of the rock specimen with the $n=0,1,2,3$ are 2689, 1911, 7352, and 6126. The existence of holes leads to the increase of the maximum acoustic emission counts produced by the rock specimens (except $n=1$ ). This is because the intact rock model has good stability and less damage before peak stress and the holes rock have poor stability and easy to produce damage before peak stress.

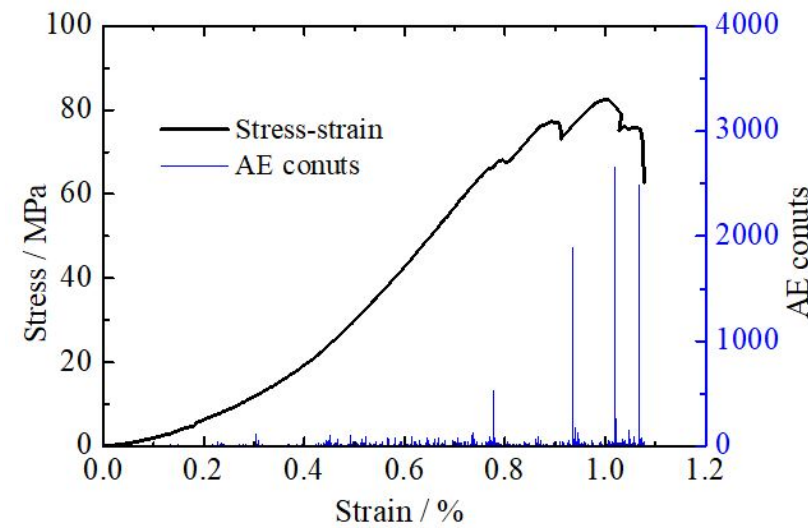

(a) $n=0$

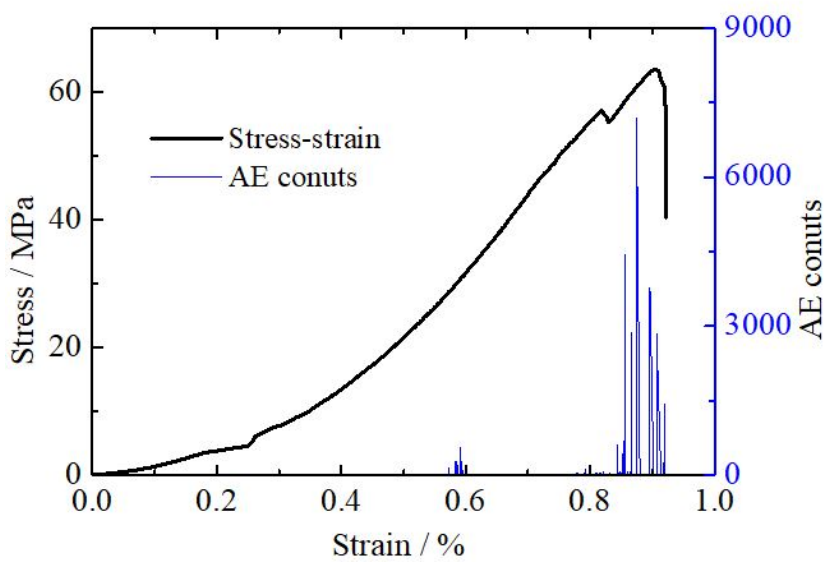

(c) $n=2$

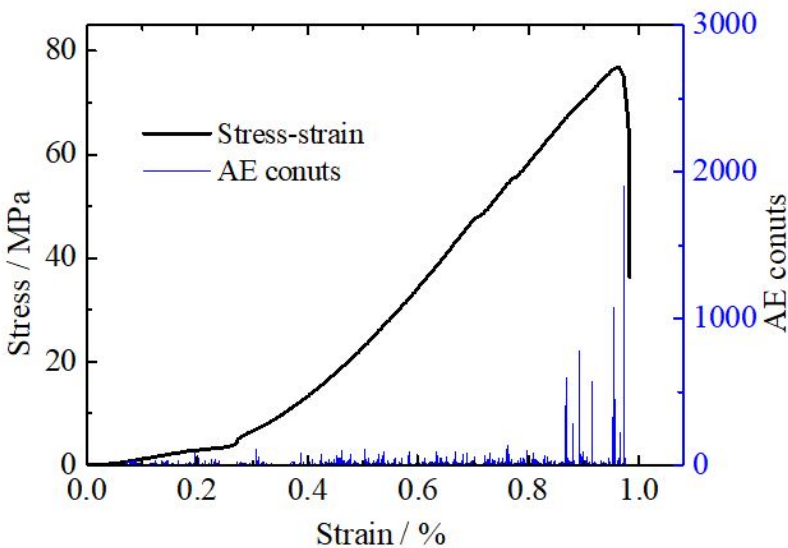

(b) $n=1$

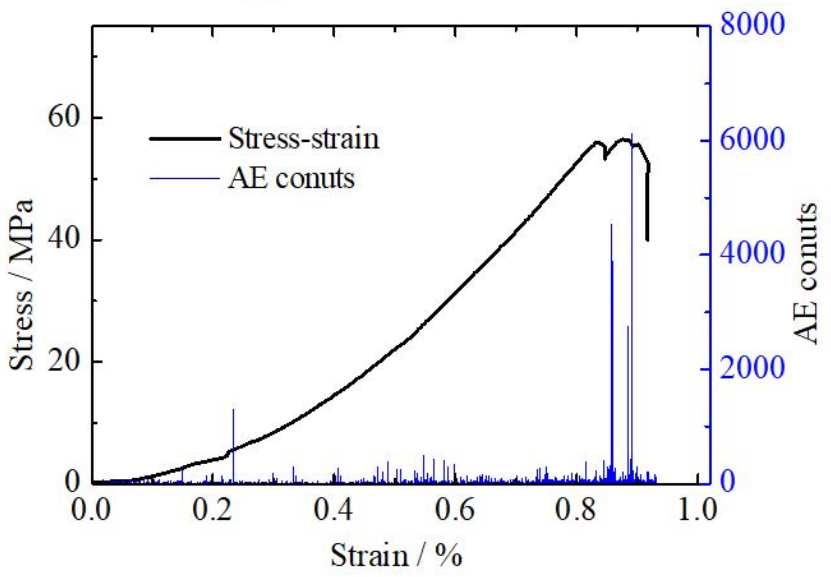

(d) $n=3$

Figure 7. Stress-AE events curves 


\section{NUMERICAL SIMULATION ANALYSIS OF SANDSTONE FAILURE}

\subsection{Numerical model establishment}

In order to further analyze the meso-mechanical characteristics of rock failure, particle discrete element numerical simulations were conducted on sandstones with different numbers of holes. The discrete element method was first proposed by Cundall et al. (Cundall and Strack, 1979) in 1979. The particle flow code(PFC) in 2 dimensions can simulate the mesoscopic failure mechanism of materials. The basic principle of PFC can refer to the literature (Itasca Consulting Group, 2008), this article will not repeat. The PFC2D numerical method has been widely used in geotechnical engineering(Wang X. and Tian L., 2018; An B. and Tannant D.D., 2007; Yoshinaka, et al. ,2008), becoming one of the effective methods for studying geotechnical engineering. Therefore, the paper uses PFC to analyze the influence of different shapes of holes on the mechanical properties of rocks from the perspective of meso-mechanics, which makes up for the shortcomings of meso-mechanical analysis in laboratory experiments.

When using PFC to solve the mechanical problems, it is always difficult to obtain the meso-mechanical parameters of the particles. At present, the stress and strain curves obtained by the simulated specimens and the indoor specimens under uniaxial compression conditions are matched and analyzed (Wang X and Tian L, 2018; An B. and Tannant D.D., 2007). When they approaching, it indicates that this set of meso-mechanical parameters is feasible. Through continuous trial and error tests, a set of meso-mechanical parameters satisfying laboratory tests was obtained, as shown in Table 1. The full stress-strain curve of the sandstone is obtained by laboratory test and numerical simulation, as shown in Fig. 8 . The main mechanical parameters are basically consistent. At the same time, the simulated specimen and the laboratory test specimen have similar failure morphology.

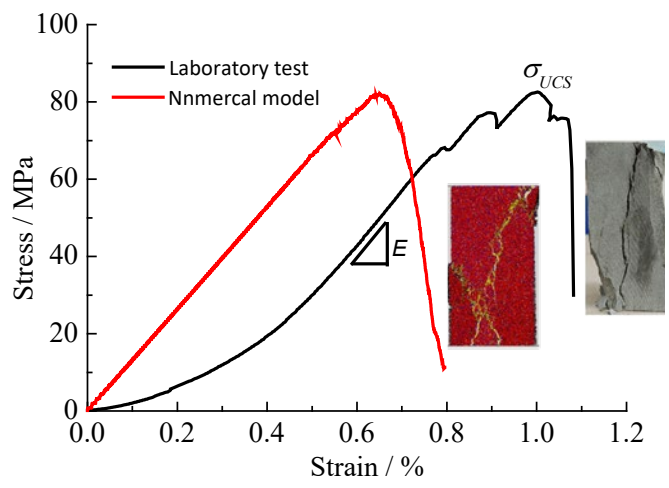

Figure 8. Numerical simulation and laboratory test stress-strain curve

Table 1. Meso-mechanical parameters of sandstone model

\begin{tabular}{cccc}
\hline Parameter & Value & Parameter & Value \\
\hline Minimum particle diameter $(\mathrm{mm})$ & 0.35 & Porosity & 0.1 \\
Particle diameter ratio & 1.6 & Bond radius multiplier & 1 \\
Density $\left(\mathrm{kg} / \mathrm{m}^{3}\right)$ & 2340 & Parallel bond tensile strength $(\mathrm{MPa})$ & 62 \\
Contact modulus of the particle $(\mathrm{GPa})$ & 8.1 & Tensile strength standard deviation & 10 \\
Parallel bond Deformation modulus $(\mathrm{GPa})$ & 8.1 & Parallel bond shear strength (MPa) & 62 \\
Friction coefficient & 0.6 & Shear strength standard deviation & 10 \\
\hline
\end{tabular}

Based on the actual rock specimen size, the PFC2D software was used to establish sandstone uniaxial compression numerical models, as shown in Fig.9. The specimen size was $50 \mathrm{~mm} \times 100 \mathrm{~mm}$, the hole radius is $3 \mathrm{~mm}$, and the distance between the holes is the same as the laboratory test specimen. 


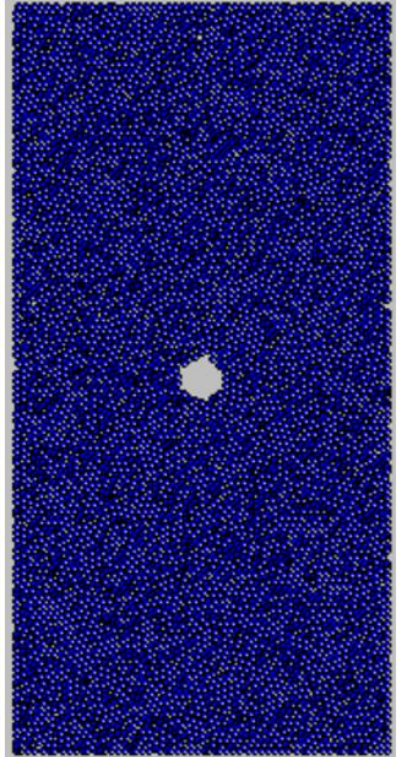

(a) $n=1$

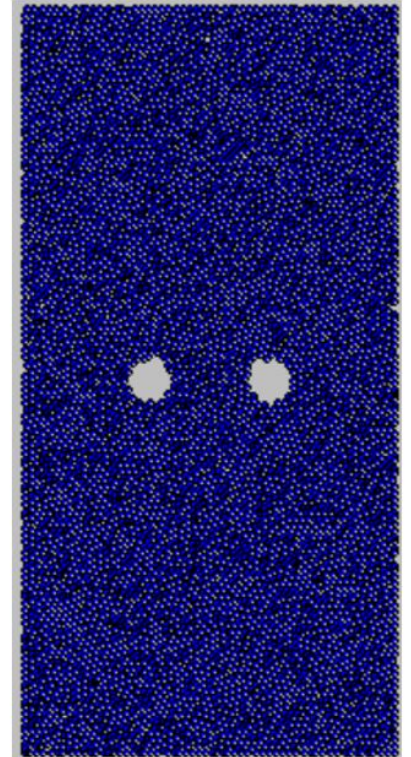

(b) $n=2$

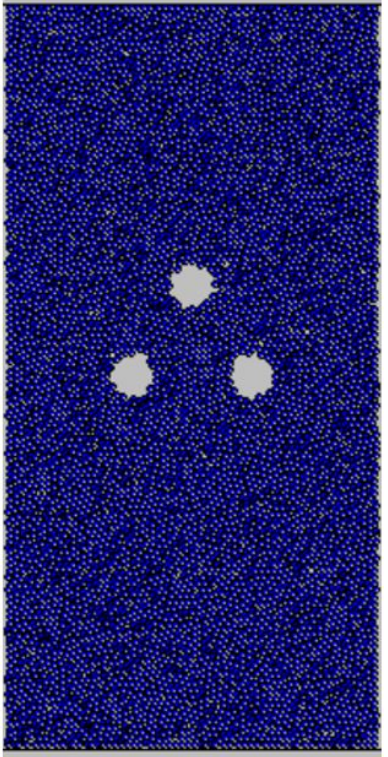

(c) $n=3$

Figure 9. Numerical models of rock with different holes

\subsection{Failure mechanical characteristic analysis}

Fig. 10 shows the stress-strain curve of the rock specimen with different number of holes. It can be seen that the shape of the stress-strain curve obtained from the numerical simulation basically agrees with the laboratory test curve. When $n$ is $0,1,2,3$, the peak stress is $82.1,74.1,63.6,56.1 \mathrm{MPa}$, respectively. Except when $n$ is 1 , the deviation is $2.75 \mathrm{MPa}$, and the others peak stress of the numerical simulation is within $1 \mathrm{MPa}$ error from the laboratory results. It also reflects the rationality of the numerical simulation.

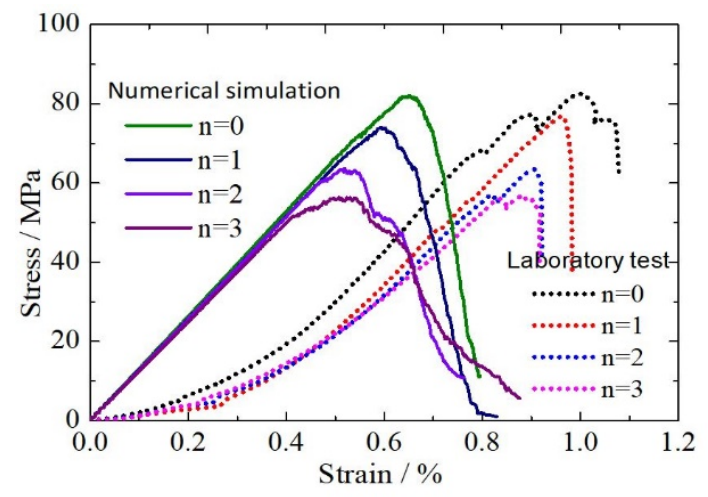

(a) Stress-strain curve

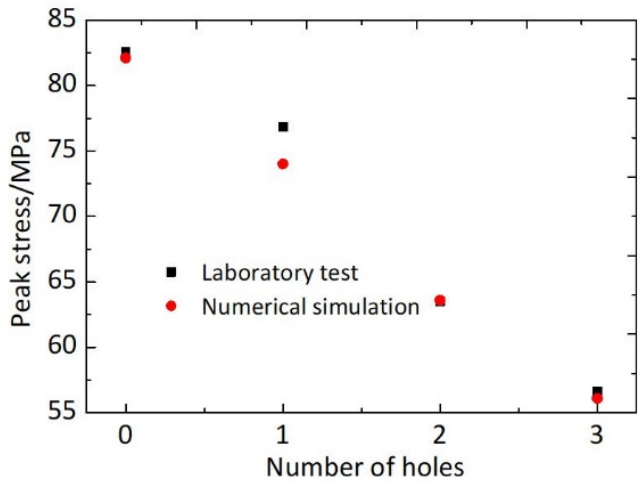

(b) Peak strength

Figure 10. Stress-strain curve and peak strength compared with laboratory test

Fig. 11 shows the failure microcrack distribution of rock specimens with different number of holes, yellow is a tensile crack and red is a shear crack. When $n$ is 1 , tensile cracks are distributed below the holes, and tensile and shear mixed cracks are distributed above the holes. Cracks first formed around the hole, and then spread along the bottom of the hole and diagonally at the upper right $45^{\circ}$, causing the specimen to failure. When $n$ is 2 , the specimen is also a composite of tensile and shear cracks. The cracks extend along the upper left of the hole on the left to form a composite crack and extend downward to form a tensile failure. The rock bridge between the holes has no cracks. The form of damage is the same as in laboratory tests. It is because the rock bridge is horizontal and perpendicular to the direction of the force. When $n$ is 3, the specimen is also composite shear and tension failure, mainly the hole on the left is damaged. The failure law is similar to that when $n$ is 2 . However, due to the increase of the holes located above, the crack also extended along the top of the hole to form a tensile failure, and the rock bridge between the left hole and the upper hole is damaged. Although there are some differences between laboratory test failure patterns and numerical simulations, there are still some similarities. 


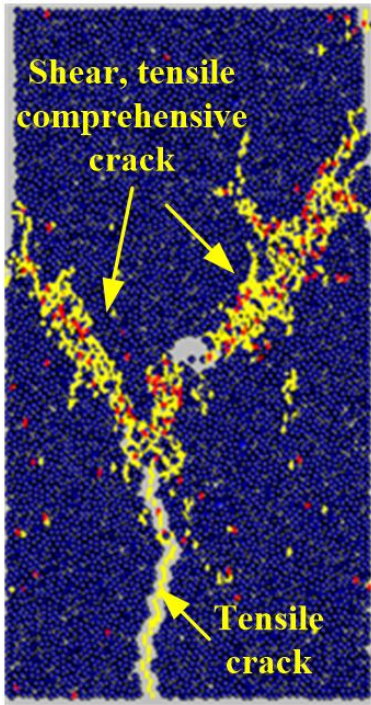

(a) $n=1$

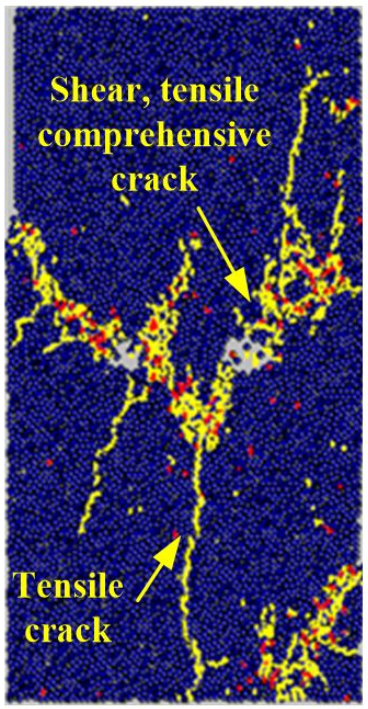

(b) $n=2$

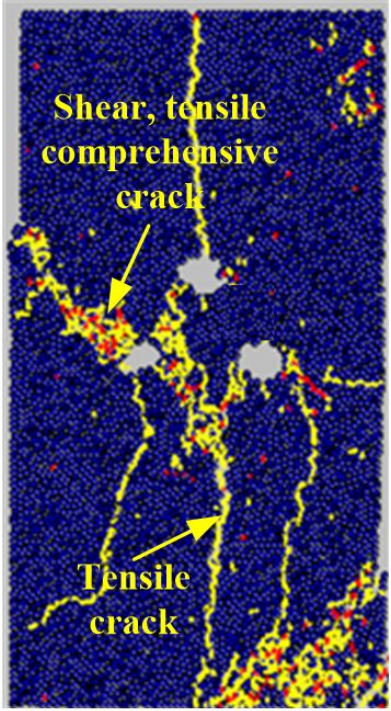

(c) $n=3$

Figure 11. Microcrack distribution of rock specimens

\section{STRESS ANALYSIS OF ROCK WITH CIRCULAR HOLE UNDER COMPRESSION}

Solving the stress of rock materials with holes has always been a difficult problem. At present, the complex variable function is mainly used to solve the problem. For some regular holes shapes, accurate analytical solutions can be obtained. For circular regular holes, elastic mechanics can be used to solve them. The mechanical model of a circular hole in compression is shown in the Fig.12. The analytical expression of stress of the hole when the model subjected to compressive stress $\sigma_{1}$ is (Erickson, M. and Durelli, A. J., 1981; Fan, H., et al, 2020):

$$
\left\{\begin{array}{l}
\sigma_{r}=\frac{\sigma_{1}}{2}\left(1-\frac{r_{1}^{2}}{r^{2}}\right)-\frac{\sigma_{1}}{2}\left(1-4 \frac{r_{1}^{2}}{r^{2}}+3 \frac{r_{1}^{4}}{r^{4}}\right) \cos 2 \theta \\
\sigma_{\theta}=\frac{\sigma_{1}}{2}\left(1+\frac{r_{1}^{2}}{r^{2}}\right)+\frac{\sigma_{1}}{2}\left(1+3 \frac{r_{1}^{4}}{r^{4}}\right) \cos 2 \theta
\end{array}\right.
$$

Where $\sigma_{r}$ is the radial stress of surrounding rock, $\sigma_{\vartheta}$ is the tangential stress of surrounding rock, $\sigma_{1}$ is the compressive stress, $r_{1}$ is the radius of the hole.

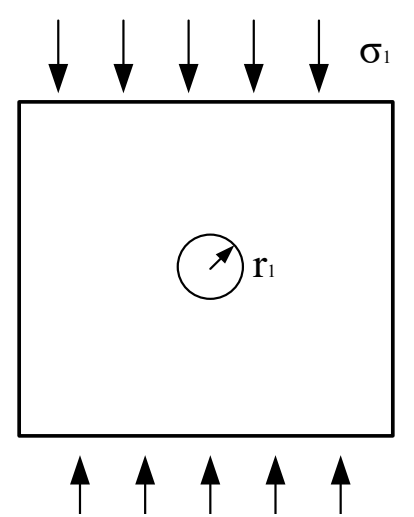

Figure 12. The mechanical model of a circular hole in compression

Through the formula (3), when $r=r_{1}$, the stress distribution expression around the hole is calculated as: 


$$
\left\{\begin{array}{l}
\sigma_{r}=0 \\
\sigma_{\theta}=\sigma_{1}+2 \sigma_{1} \cos 2 \theta
\end{array}\right.
$$

A schematic diagram of the stress distribution around the circular hole wall is shown in Fig. 13.

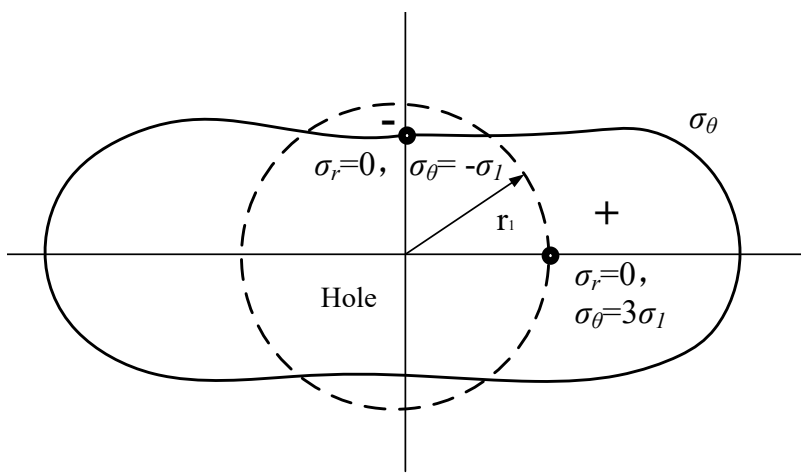

Figure 13. Schematic diagram of stress distribution around hole wall

It can be seen from the formula (4) that when $\theta=0^{\circ}$ and $r=r_{1}$, the radial stress and the tangential stress are $\sigma_{r}=0, \sigma_{\vartheta}=3 \sigma_{1}$, respectively. When $\vartheta=90^{\circ}$ and $r=r_{1}$, the radial stress and the tangential stress are $\sigma_{r}=0, \sigma_{\vartheta}=-\sigma_{1}$, respectively. The compressive stress concentration occurs on the left and right sides of the circular hole, and the tensile stress concentration occurs at the top and bottom boundary of the hole. The failure of the models first occurs at these stress concentrations where microcracks are produced. Then the crack expands and eventually breaks.

Fig. 14 is a distribution of microcracks around a circular hole, where yellow is a tensile crack and red is a shear crack. It can be seen from Fig. 14 that the tensile crack occurs at the top and bottom of the hole, mainly because the top and bottom positions are affected by tensile stress. Tensile and shear mixed cracks appear on the left and right sides of the hole, mainly because the external force exceeds the bond strength between the particles, so tension and shear cracks appear. This is also the principle of compression-induced tensile shear failure (Itasca Consulting Group, 2008). It can be seen that the crack distribution of numerical simulation is consistent with the theoretical stress analysis results. It also reflects the correctness of numerical simulation.

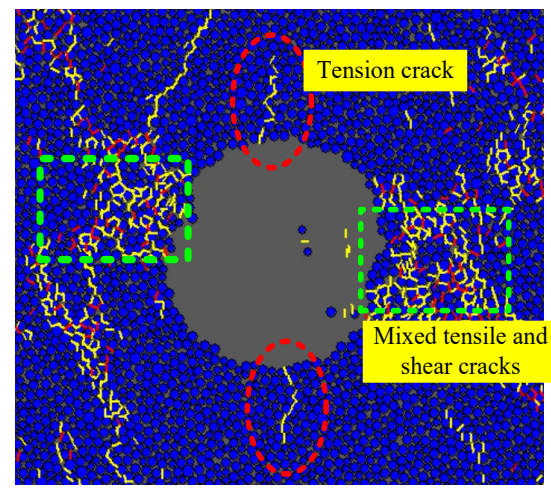

Figure 14. Crack distribution around the hole

\section{CONSTITUTIVE MODEL OF ROCK DAMAGE}

\subsection{Rock damage variables based on AE characteristics}

$\mathrm{AE}$ can evaluate the damage of the rock mass. In order to further quantitatively analyze the damage of the rock mass. The damage variable is used to study the damage of the rock mass. Currently, there are many parameters to define damage variables, such as cracks, the elastic coefficient, the yield stress, the density, and the AE characteristics (Aggelis., 2011; Kupchella et al., 2015; Murti et al., 1991; Liu et al., 2019b; Guo, Q., et al., 2020b). Therefore, this paper uses the number of $A E$ events to define the damage variable.

Using this parameter, the damage variable $D$ can be defined as: 
$D=\frac{A_{d}}{A}$

Where $A_{d}$ is the total number of $\mathrm{AE}$ counts from a rock specimen in a certain amount of time, $A$ is the total number of $A E$ counts.

Fig. 15 show the damage variable of rock based on the AE characteristics. According to the graph, the damage evolution of rock can also be divided into four parts, including stable damage stage, slowly increasing damage stage, sharply increasing damage stage and stable stage. In the stable damage stage, the holes has little effect because there are no damage or few damage cracks in the rock. However, the holes has a great influence on the slowly increasing damage stage and sharply increasing damage stage. In the stage of damage slow increase, the damage variable increases most rapidly when $n=1$, followed by $n=3$ and $n=2$, and the slowest increase is $n=0$. The reason is that the mechanical properties of rock without hole is better, their compressive capacity is stronger, and it is not easy to be damaged under lower stress intensity. In the stage of damage rapid increase, the effect of holes on the damage of rock is opposite to that of damage slow increase stage. When $n=1$, the increase of damage variable is the smallest, followed by $n=0$, and the fastest is $n=2$ and 3 . The reason is that under this condition, a large number of cracks are produced in the slow damage stage of the holes rock and when the rock enters the stage of rapid damage increase, the damage crack propagation induces the large-scale damage of the rock. In the stable stage, the damage variable value eventually approaches 1.

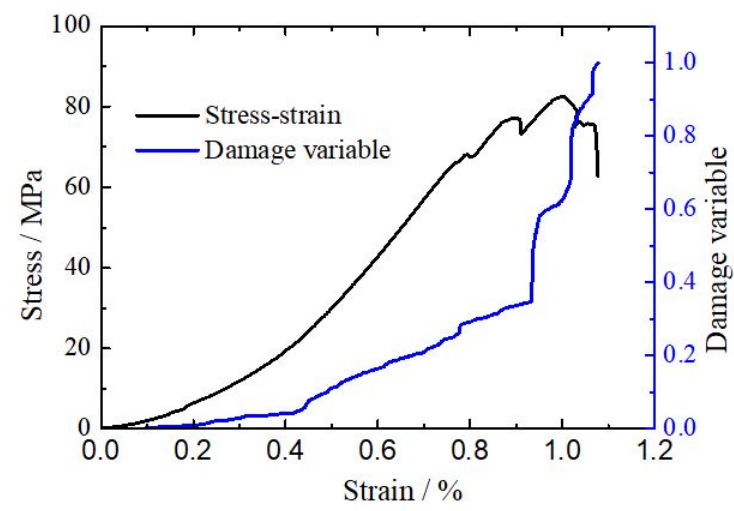

(a) $n=0$

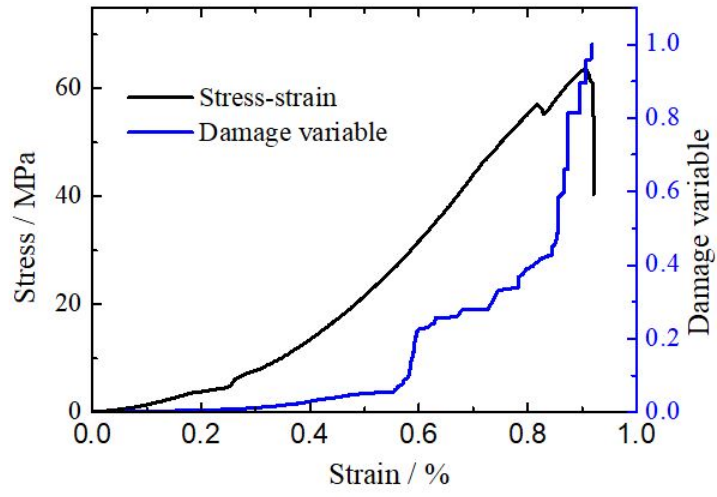

(c) $n=2$

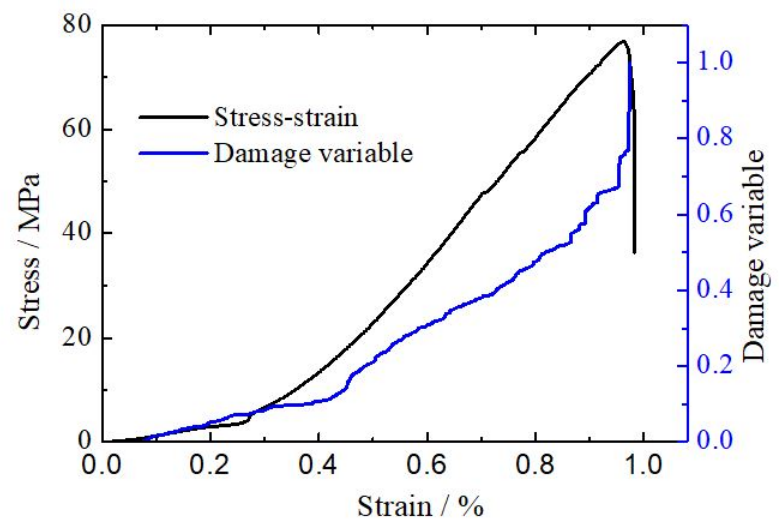

(b) $n=1$

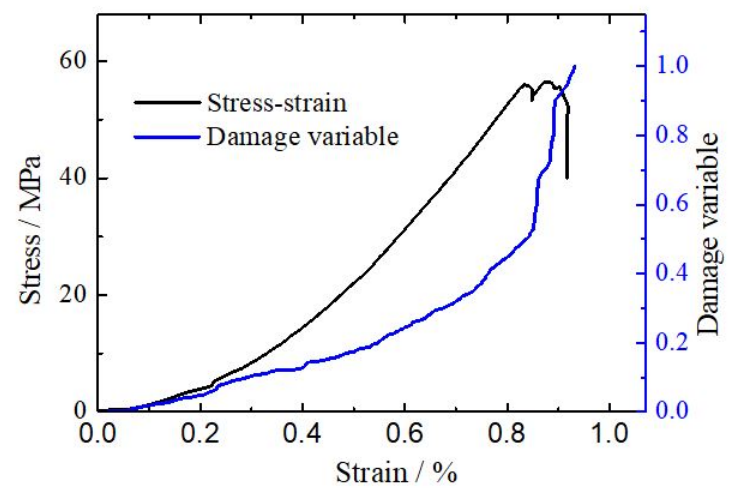

(d) $n=3$

Figure 15. Damage evolution characteristics of rock based on the damage variable

\subsection{Rock Damage Constitutive Model}

The rock constitutive model has always been one of the hot and difficult issues in rock mechanics research. In 1970 , Duncan proposed a Duncan model for describing nonlinear elastic constitutive properties of geotechnical materials (Duncan and Chang, 1970). The expression is as follows:

$\sigma=\frac{\varepsilon}{a+b \varepsilon}$ 
The Duncan model is simple in form and does not require artificial setting of the yield point. Various parameters can be determined through experiments, and its parameters have physical meanings related to rock properties. However, the model only reflects the linear elasticity and strain hardening stages of the rock, and cannot describe the initial compaction stage of the geotechnical material. Therefore, based on the Duncan model, a quadratic term is added so that it can better reflect the initial-compaction stage of the rock and better reflect the stress-strain relationship of rock materials. Therefore, the improved Duncan model equation is as follows (Jiang, Y., et al. 2005):

$$
\begin{aligned}
& \sigma=\frac{\varepsilon}{a+b \varepsilon+c \varepsilon^{2}} \\
& a=1 / E_{0}, b=1 / \sigma_{c}-2 / \varepsilon_{c} E_{0}, c=1 / E_{0} \varepsilon_{c}^{2}
\end{aligned}
$$

Where $E_{0}$ is the initial elastic modulus of the $\operatorname{rock}\left(E_{0}=\mathrm{d} \sigma / \mathrm{d} \varepsilon \mid \varepsilon_{\varepsilon \rightarrow 0}\right), \sigma_{c}$ is the peak strength of the rock, $\varepsilon_{c}$ is the axial strain corresponding to the peak strength, and $c$ is the fitting parameter related to the rock.

Eq. (7) can only reflect the stress-strain constitutive relationship the pre-peak of the rock, but cannot reflect the post-peak strain softening stage. In 1999, Lemaitre et al. (1999) proposed the principle of equivalent strain. Based on previous studies, the article introduces damage variables, the damaged constitutive model Eq. of the rock is established as:

$$
\sigma=\frac{\varepsilon}{a+b \varepsilon+c \varepsilon^{2}}(1-D)
$$

Bringing Eq. (5) into Eq. (9) gives the rock damage constitutive model Eq.

$$
\sigma=\frac{\varepsilon}{a+b \varepsilon+c \varepsilon^{2}}\left(1-\frac{A_{d}}{A}\right)
$$

Fig. 16 shows the stress-strain curves of test result and fitting result according to Eq. (10) with different $n$. It can be seen that the stress-strain curves tested are in good agreement with the stress-strain curves obtained from Eq. (10). No matter it is the pre-peak stage or the post-peak stage can all agree well with the test curve. Make up for the shortcomings of Eqs. (6) and (7). And the acoustic emission is easy to monitor during the test. Therefore, Eq. (10) can be used to represent the damage constitutive model of rocks. It can be known from Tab.1 that when the $n$ is $0,1,2$, and 3, the fitting accuracy values $R$ is $0.9988,0.9956,0.9980$, and 0.9987 , respectively. It can be seen that the fitting accuracy values are all above 0.99. Therefore, the damage constitutive model of rock can be well represented by Eq. (10).

It can be known from Table 2 that when $n$ is $0,1,2$ and 3, the values of parameter $a$ is $0.04423,0.06801,0.07167$, and 0.07194 , respectively. As $n$ increases, the value of $a$ also increases. According to Eq. (8), it can be known that $a$ is related to the initial elastic modulus $E_{0}$, and the value of $E_{0}$ decreases as $n$ increases. When $n$ is $0,1,2$ and 3, the values of parameter $b$ is $-0.07848,-0.1265,-0.1394$, and -0.1403 , respectively. As $n$ increases, the value of $b$ decreases. The value of $b$ is mainly related to the peak strength, initial elastic modulus and peak strain. When $n$ is $0,1,2$ and 3 , the values of parameter $c$ is $0.04717,0.07261,0.08480$, and 0.08557 , respectively. As $n$ increases, the value of $c$ increases. The value of $c$ is mainly related to the initial elastic modulus and peak strain. When $\mathrm{n}$ goes from 0 to 1 , the value of $a, b$, and $c$ changes greatly, and when $\mathrm{n}$ goes from 1 to 3 , the value of $a, b$, and $c$ changes little. Mainly because of the existence of holes, the mechanical properties of rocks have changed. 


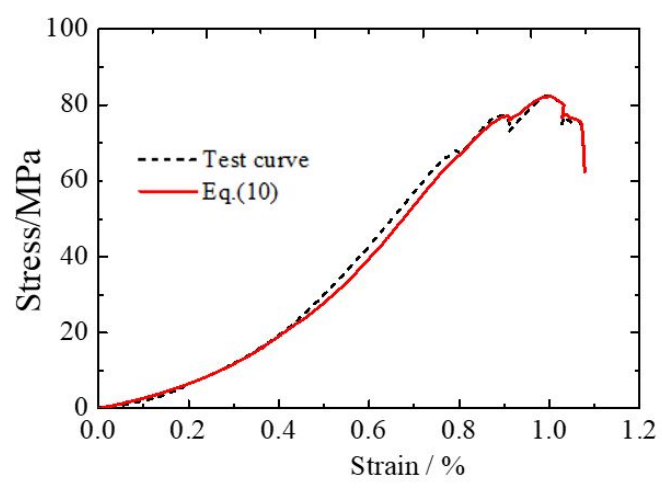

$n=0$

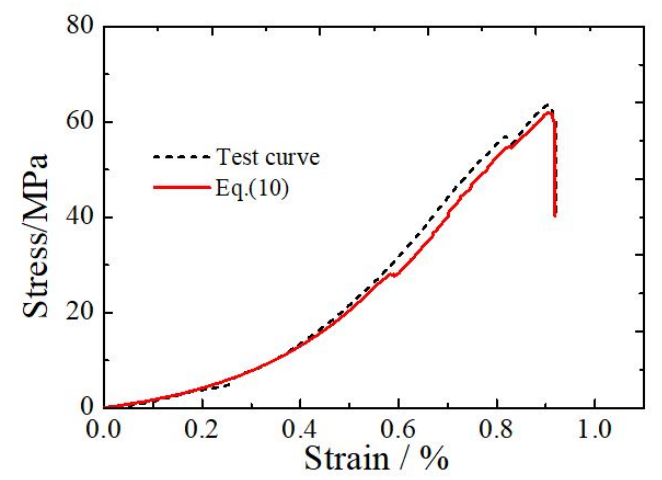

$n=2$
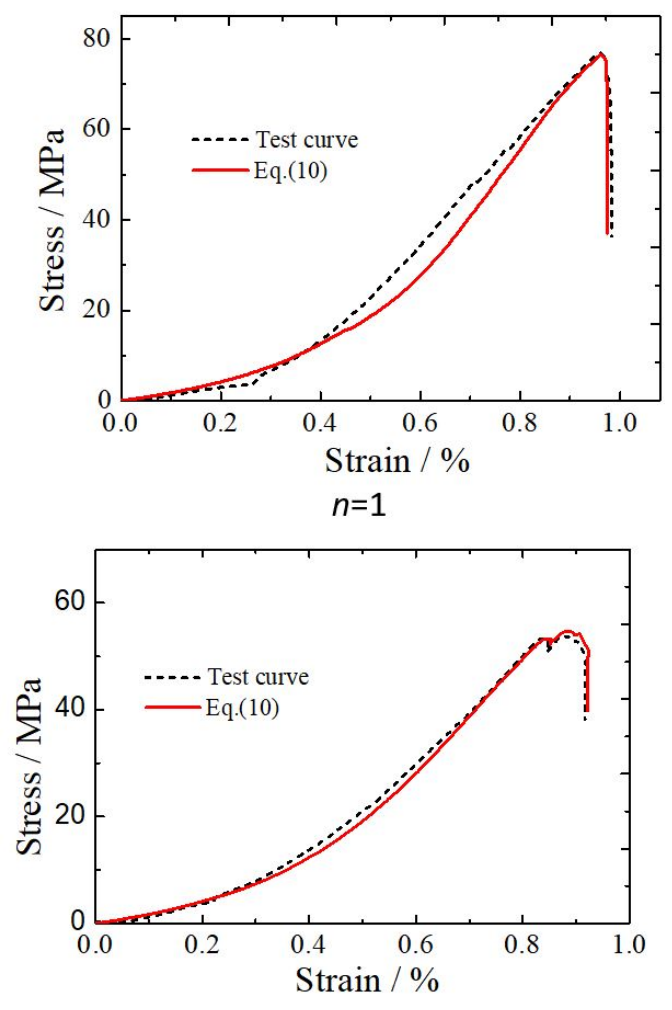

$n=3$

Figure 16. Fitting Stress-strain curves of rock with different number of holes

Table 2. Values of parameters a, b, c and fitting accuracy

\begin{tabular}{ccccc}
\hline specimen & $\mathbf{n = 0}$ (intact) & $\boldsymbol{n = 1}$ & $\boldsymbol{n = 2}$ & $\mathbf{n = 3}$ \\
\hline $\mathrm{a}$ & 0.04423 & 0.06801 & 0.07167 & 0.07194 \\
$\mathrm{~b}$ & -0.07848 & -0.1265 & -0.1394 & -0.1403 \\
$\mathrm{c}$ & 0.04717 & 0.07261 & 0.08480 & 0.08557 \\
$\mathrm{R}$ & 0.9988 & 0.9956 & 0.9980 & 0.9987 \\
\hline
\end{tabular}

\section{CONCLUSION}

This paper conducts uniaxial compression tests on rocks with different numbers of holes. Firstly, the effect of holes on the mechanical characteristics of rock failure is analyzed, then the AE characteristics of rock with holes are analyzed, the meso-mechanical characteristics of rock failure are analyzed by numerical simulation, and finally the constitutive model of rock with holes is analyzed. The conclusions are as follows.

1. As the number of holes increases, the elastic modulus and peak strength of the rock decrease, and the strength of the rock weakens significantly. The decreasing degree of peak strength and elastic modulus of rock is approximately one-dimensional linear relationship with the $n$. The stress-strain curves can be divided into 4 stages. They are initial compaction stage, elastic stage, strain hardening stage, and post-peak strain softening stage. The AE counts gone through stable, slow increase, a sudden increase, and towards zero four stages and these four stages are corresponding to the four stages of the stress-strain curves approximately.

2. Theoretical analysis of stress distribution characteristics around the rock with circular holes is carried out. The compressive stress concentration occurs on the left and right sides of the circular hole, the tensile stress concentration occurs at the top and bottom boundary of the hole. And the tensile crack occurs at the top and bottom of the hole, tensile and shear mixed cracks appear on the left and right sides of the hole from numerical simulation. The crack distribution of numerical simulation is consistent with the theoretical stress analysis results.

3. According to Duncan model, the constitutive model was established in consideration of rock damage. No matter it is the pre-peak stages or the post-peak stage, the fitting curves are in good agreement with the test curves. It also 
illustrates the rationality of the damage constitutive model. This article is of great significance to understand the rock failure mechanics and damage characteristics.

Author's Contribuitions: Conceptualization, W Liu; Methodology, C Peng and W Liu; Formal analysis, C Peng; Validation, W Liu; Writing - original draft, C Peng and W Liu; Writing - review \& editing, C Peng and W Liu.

Editor: Rogério José Marczak.

\section{References}

An, B., Tannant, D.D. (2007). Discrete element method contact model for dynamic simulation of inelastic rock impact. Computer and Geosciences 33:513-521.

Aggelis, D.G. (2011). Classification of cracking mode in concrete by acoustic emission parameters. Mechanics Research Communications 38(3): 153-157.

Cundall, P.A., Strack, O.D.L., (1979). A discrete numerical model for granular assemblies. Geotechnique 29:331-336.

Duncan, J.M., and Chang, C.Y. (1970). Nonlinear analysis of stress and strain in soils. Journal of the Soil Mechanics and Foundations 96(5):1629-1654.

Erickson, M., \& Durelli, A. J. (1981). Stress distribution around a circular hole in square plates, loaded uniformly in the plane, on two opposite sides of the square. Journal of Applied Mechanics 48: 203-204.

Fan, H., Wang, L., Liu, W. (2020). An analytical solution for stresses and deformations of tunnels in a non-uniform stress field based on strain-softening model and Mogi-Coulomb criterion. Latin American Journal of Solids and Structures 17: 1-16.

Fakhimi, A., Gharahbagh, E.A. (2011). Discrete element analysis of the effect of pore size and pore distribution on the mechanical behavior of rock. International Journal of Rock Mechanics and Mining Sciences 48(1): 77-85.

Guo, Q., Pan, J., Cai, M., et al. (2020a). Investigating the Effect of Rock Bridge on the Stability of Locked Section Slopes by the Direct Shear Test and Acoustic Emission Technique. Sensors 20(3): 638.

Guo, Q., Pan, J., Cai, M., et al. (2020b). Analysis of Progressive Failure Mechanism of Rock Slope with Locked Section Based on Energy Theory. Energies 13(5): 1128.

Hirata, A., Kameoka, Y., Hirano, T. (2007). Safety management based on detection of possible rock bursts by AE monitoring during tunnel excavation. Rock Mechanics and Rock Engineering 40(6):563-576.

Huang. Y.H., Yang, S.Q., Ranjith, P.G., Zhao, J. (2017). Strength failure behavior and crack evolution mechanism of granite containing pre-existing non-coplanar holes: experimental study and particle flow modeling. Computers and Geotechnics 88:182-198.

Jiang, Y., Xian, X., Jian, S. (2005). Research on distortion of single rock and constitutive relation. Rock and Soil Mechanics(in Chinese) 26(6): 941-945.

Kim, J.S., Lee, K.S., Cho, W.J., Choi, H.J., Cho, G.C. (2015). A comparative evaluation of stress-strain and acoustic emission methods for quantitative damage assessments of brittle rock. Rock Mechanics and Rock Engineering 48(2): 495-508.

Kupchella, R., Stowe, D., Xiao, X., Algoso, A., Cogar, J. (2015). Incorporation of material variability in the Johnson Cook model. Procedia Engineering 103: 318-325.

Lemaitre, J., Sermage, J.P., Desmorat, R. (1999). A two scale damage concept applied to fatigue. International Journal of Fracture 97:67-81.

Lockner, D. (1993). The role of acoustic emission in the study of rock fracture. International Journal of Rock Mechanics and Mining Sciences \& Geomechanics Abstracts 30(7): 883-899.

Lin, P., Wong, R.H., Tang, C.A. (2015). Experimental study of coalescence mechanisms and failure under uniaxial compression of granite containing multiple holes. International journal of rock mechanics and mining sciences 77:313-327. 
Liu, Q., Xu, J., Liu, X. (2015). The role of flaws on crack growth in rocklike material assessed by AE technique. International Journal of Fracture 193(2):99-115.

Liu, K., Ostadhassan, M. (2017). Multi-scale fractal analysis of pores in shale rocks. Journal of Applied Geophysics 140: 1-10.

Liu, W.R., Liu, J.K., Zhu, C. (2019a). Multi-scale effect of acoustic emission characteristics of 3D rock damage. Arabian Journal of Geosciences 12(22), 668.

Liu, W., Yuan, W., Yan, Y., Wang, X. (2019b). Analysis of Acoustic Emission Characteristics and Damage Constitutive Model of Coal-Rock Combined Body Based on Particle Flow Code. Symmetry 11(8), 1040.

Itasca Consulting Group (2008) PFC2D (particle flow code in 2 dimensions) users guide. Itasca, Minneapolis

Moradian, Z.A., Ballivy, G., Rivard, P., Gravel, C., Rousseau, B. (2010). Evaluating damage during shear tests of rock joints using acoustic emissions. International Journal of Rock Mechanics and Mining Sciences 47(4): 590-598.

Murti, V., Zhang, W., Valliappan, S. (1991). Stress invariants in an orthotropic damage space. Engineering fracture mechanics 40(6): 985-990.

Wang, S.Y., Sloan, S.W., Fityus, S.G., Griffiths, D.V., Tang, C.A. (2013). Numerical modeling of pore pressure influence on fracture evolution in brittle heterogeneous rocks. Rock mechanics and rock engineering 46(5):1165-1182.

Wasantha, P.L., Ranjith, P.G., Shao, S.S. (2014). Energy monitoring and analysis during deformation of bedded-sandstone: use of acoustic emission. Ultrasonics 54(1): 217-226.

Wang, X., Tian, L. (2018). Mechanical and crack evolution characteristics of coal-rock under different fracture-hole conditions: a numerical study based on particle flow code. Environmental Earth Sciences 77(8): 297.

Wang, X., Wen, Z., Jiang, Y., Huang, H. (2018). Experimental study on mechanical and acoustic emission characteristics of rocklike material under non-uniformly distributed loads. Rock Mechanics and Rock Engineering 51(3): 729-745.

Yoshinaka, R., Osada, M., Park, H., et al. (2008). Practical determination of mechanical design parameters of intact rock considering scale effect. Engineering Geology 96: 173-186. 